\title{
Advances in the prevention of mother-to-child transmission of HIV and resulting clinical and programmatic implications
}

REVIEW

This article was published in the following Dove Press journal:

Research and Reports in Neonatology

10 July 2014

Number of times this article has been viewed

\author{
Shrinivas Darak ${ }^{1,2}$ \\ Ritu Parchure 2 \\ Trupti Darak ${ }^{2}$ \\ Rachel Talavlikar ${ }^{3}$ \\ Sanjeevani Kulkarni \\ Vinay Kulkarni \\ 'Population Research Centre, \\ Faculty of Spatial Sciences, University \\ of Groningen, the Netherlands; \\ 2PRAYAS Health Group, Pune, India; \\ ${ }^{3}$ Department of Family Medicine, \\ University of Calgary, Calgary, \\ $A B$, Canada
}

\begin{abstract}
Following scientific advancements in the prevention of mother-to-child transmission (PMTCT) of HIV, a global plan is being implemented to virtually eliminate new pediatric HIV infections. However, despite revisions to guidelines for providing antiretroviral medications, including the availability of more effective drug regimens over the past decade, and the recent rapid scale-up of PMTCT services, sizeable challenges persist in achieving this goal. This paper provides a summary of recent approaches to the provision of antiretroviral medicines to pregnant women and the resulting implications. Emerging programmatic and service delivery considerations, aiming to increase the effectiveness of PMTCT programs to overcome barriers faced by women in accessing PMTCT services are also discussed. Worldwide elimination of pediatric HIV is now a possibility due to the increasing similarity of PMTCT guidelines in developing countries to those in developed countries. However the resulting practical challenges of implementation will require resources and support at local, national, and international levels. As developing countries strive to adopt and implement more efficacious drug regimens, the effectiveness of PMTCT programs will be determined by coverage of services and the recruitment and retention of pregnant women in the cascade. To expand services and provide an effective, comprehensive approach in under resourced settings, harmonized efforts to address barriers whilst considering contextual factors will be needed.
\end{abstract}

Keywords: PMTCT, HIV, ARVs, program implementation

\section{Introduction}

Since the breakthrough clinical trial (ACTG 076) that demonstrated the effectiveness of antiretroviral medicines (ARVs) for the prevention of mother-to-child transmission of HIV (PMTCT), ${ }^{1}$ numerous clinical trials have been conducted to evaluate the efficacy of ARVs for PMTCT in different combinations administered for varied durations. ${ }^{2}$ With current regimens, mother-to-child transmission of HIV (MTCT) can be reduced to less than $2 \%$ from a possible $25 \%-35 \%$ (in the absence of any intervention). ${ }^{3}$

The discovery of efficacious antiretroviral regimens for PMTCT was an important landmark in the AIDS epidemic. Following scientific achievements, in 2009 the United Nations, along with its partners, embarked to achieve the goals of virtually eliminating MTCT (eMTCT) from the world and keeping HIV-infected mothers alive. ${ }^{4}$ The new Global Plan aims by 2015 to reduce HIV infections among children by $90 \%$ and AIDS-related maternal deaths by $50 \% .^{5}$ The path to achieve these goals however is fraught with several challenges.

A comprehensive four prong approach for implementation of PMTCT programs established by the United Nations in 2002 was again espoused in the new plan
Correspondence: Shrinivas Darak Population Research Centre, Faculty of Spatial Sciences, University of Groningen, Landleven I, 9747 AD, Groningen, the Netherlands $\mathrm{Tel}+3$ I 50363388 I

Emails.darak@rug.nl; shirishdarak@ gmail.com
Dovepress

http://dx.doi.org/1 $0.2147 /$ RRN.S46237
Research and Reports in Neonatology 20 I4:4 I I I-I23

(c) (1) (2) ( 2014 Darak et al. This work is published by Dove Medical Press Limited, and licensed under Creative Commons Attribution - Non Commercial (unported, v3.0) 
for eMTCT. ${ }^{6}$ It includes: 1) prevention of HIV in women of reproductive age; 2 ) prevention of unintended pregnancies in women with HIV; 3 ) prevention of HIV transmission from mother-to-child; and 4) provision of ongoing care and support to mothers, their children, and families. The most important challenge is the implementation of a comprehensive approach to PMTCT to achieve universal coverage of PMTCT services. Traditionally, PMTCT programs worldwide have focused on the identification of HIV infection among pregnant women and on the provision of medicines to the mother-baby pair to prevent HIV transmission (Prong 3 ) rather than implementing a comprehensive approach. ${ }^{7}$

In spite of the recent rapid scale-up of services for PMTCT, as of December 2012, only $62 \%$ of HIV-infected pregnant women receive effective (excluding single dose nevirapine $[\mathrm{NVP}]$ ) antiretroviral prophylaxis or treatment. ${ }^{8}$ While the rates of MTCT have been declining, there are significant new HIV infections occurring among children. Despite increased coverage, 260,000 (range: 230,000-320,000) children were vertically infected with HIV in $2012 .^{8}$

Studies have shown that in order to achieve the elimination of new child infections, PMTCT programs must achieve high coverage of effective ARV interventions and safer infant feeding practices. ${ }^{69}$ Based on these studies, a relationship between the effectiveness of ARV interventions for PMTCT and coverage of these interventions to achieve reduction in MTCT can be shown diagrammatically. Figure 1 is a visual representation of the relationship between the effectiveness of PMTCT protocols and program coverage to achieve a reduction in MTCT rates. The virtual elimination of pediatric HIV is not possible with less effective drug protocols, such as single dose NVP, even if high coverage of services are offered. It will be possible only with high coverage of effective PMTCT protocols and programs.

Until recently scientific developments have focused on identifying effective ARVs for reducing MTCT and have formed the basis for informing PMTCT protocols. However, the recent World Health Organization (WHO) recommendation (2010) for low and middle income countries (LMIC) emphasizes the importance of the simplicity, as well as harmonization of these protocols for effective delivery. Programs are now expected to help mitigate the individual, societal and health systems related factors that act as barriers to accessing PMTCT services.

This article provides a summary of current guidelines for PMTCT, including highlights of recent changes, to approaches and their resulting implications. Potential shortand long-term effects of these changes as well as challenges that will need to be overcome to implement services (in under resourced settings in particular) are discussed. Emerging program and service delivery considerations that aim to increase the effectiveness of PMTCT programs are also explored.

PMTCT guidelines are summarized based on documents of respective agencies. MEDLINE database was searched for published research articles regarding clinical implications of PMTCT guidelines by searching for specific topics such

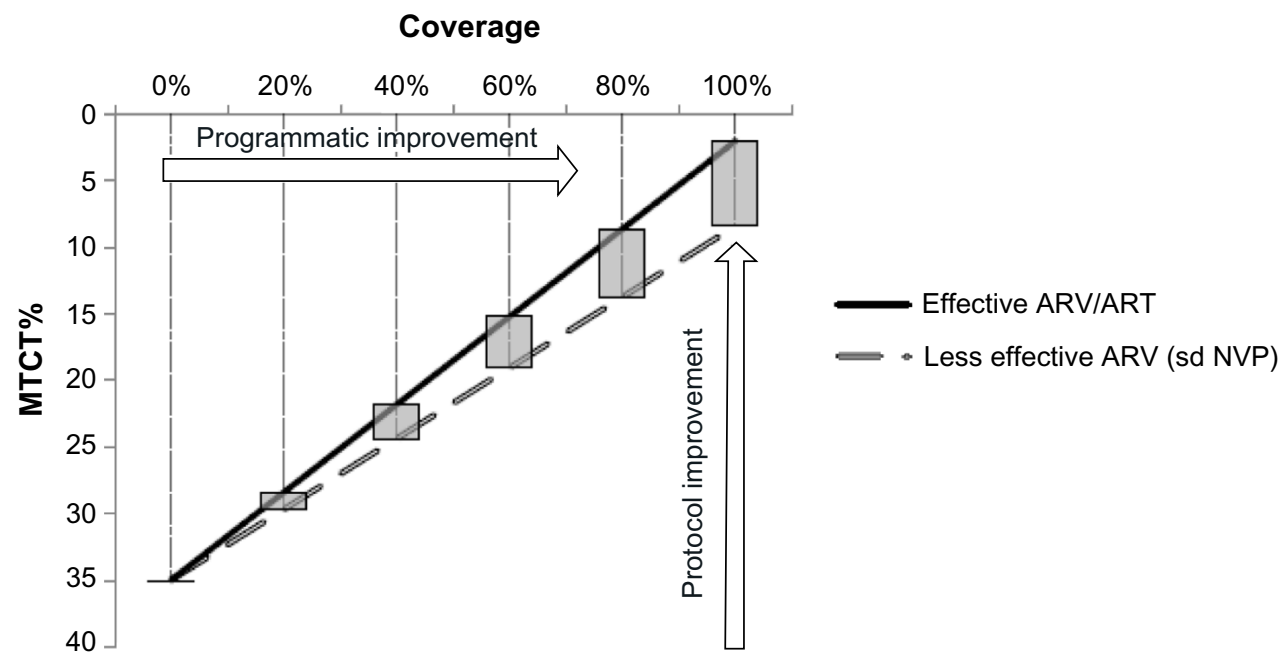

Figure I Visual representation of the relationship between the efficacy and coverage of PMTCT services.

Notes: This figure is not based on any mathematical modeling. The arrows in the figure demonstrate two dimensions through which PMTCT can be achieved. The vertical arrow signifies transitioning of the PMTCT programs from less effective to more effective PMTCT drug regimens (from sd NVP to Option A, Option B, or Option B+). The horizontal arrow indicates program coverage which can be measured by calculating the number of women completing the cascade of PMTCT services. HIV transmission rate of $2 \%$ for effective ARVIART and $8.4 \%$ for less effective ARV (sd NVP as per HIVNET-0I2 trial) were assumed for making the comparison.

Abbreviations: MTCT, mother-to-child transmission of HIV; PMTCT, prevention of mother-to-child transmission; NVP, nevirapine; ARV, antiretroviral medicine; ART, antiretroviral therapy; sd, single dose. 
as maternal mortality, drug resistance among women and children, and pregnancy outcomes. Basing our summary on implications for service delivery, we searched for recently published (after 2012) systematic reviews that addressed barriers and facilitators to providing PMTCT services as well as programmatic approaches for delivering services.

\section{Comparison of current PMTCT guidelines}

The science of PMTCT has evolved rapidly in the past decade. Studies have shown that compared to mono- or dual-therapy ARV regimens, the use of triple ARVs is more efficacious in reducing MTCT. ${ }^{10}$ It is also evident that ARV during breastfeeding $(\mathrm{BF})$ reduces the risk of transmission through breast milk. ${ }^{11}$

Keeping pace with evidence from clinical trials, there have been major changes in recommendations for PMTCT in the past decade. Various public health agencies such as the WHO, British HIV Association (BHIVA) and the US Department of Health and Human Services (US DHHS) each issue updated PMTCT guidelines regularly. Most nations from developing regions follow the WHO guidelines aimed at low and middle income countries. ${ }^{12}$ Guidelines developed by DHHS, WHO European regional office, and BHIVA are aimed at specific developed regions such as the USA, Europe and UK respectively. Other developed countries such as Canada and Australia base their recommendations on DHHS guidelines.

The comparative differences and similarities across some major guidelines related to essential steps in delivery of PMTCT services are summarized in Table 1. All major guidelines now recommend the use of combination ARVs (cARV) in HIV-infected women for PMTCT. However, there are still some differences in terms of choice of ARV medicines and infant feeding guidelines.

\section{HIV testing}

Evidence from both resource-rich and resource-poor settings indicates that the uptake of HIV testing increases when it is routinely discussed and offered, and where it is wellintegrated into prenatal care. ${ }^{13} \mathrm{WHO}$ guidelines for LMIC recommend provider initiated HIV testing and counseling for pregnant women. Repeat testing in the third trimester, during labor, or shortly after delivery is recommended in all settings with a generalized epidemic. In provider initiated testing, health care providers recommend HIV testing to patients attending health facilities after providing sufficient information to make an informed and voluntary decision to be tested. It also entails maintaining patient confidentiality, performing posttest counseling and making referrals to appropriate services. ${ }^{13}$

The guidelines from most developed countries have adopted the policy of universal HIV testing as a part of routine prenatal care, with patient notification (opt-out screening). There are a few differences as far as repeat testing is concerned. DHHS guidelines recommend it only in jurisdictions with elevated rates of HIV infection among pregnant women, whilst BHIVA guidelines recommend it in women with continued risk of infection.

\section{Eligibility criteria for initiating antiretroviral} therapy (ART) for mother's own health

Until the 2010 WHO guidelines for LMIC, guidelines recommended a lower CD4 cut off value $(200 / \mathrm{cmm})$ as compared to other developed country guidelines. ${ }^{14,15}$ However, current WHO recommendations for LMIC are either to initiate ART at CD4 cut off of 500/cmm or initiate treatment for all pregnant and BF women. This approach is not only aimed at eliminating MTCT but is also expected to ensure better health in mothers and reduce sexual transmission. ${ }^{12}$ In contrast, guidelines for the $\mathrm{UK}^{16}$ and Europe ${ }^{17}$ maintain CD4 cut off values of $350 / \mathrm{cmm}$ to initiate ART. DHHS guidelines recommend initiating ART in all HIV-infected individuals. $^{18}$

The question of whether to initiate ART during the first trimester in eligible pregnant women has been especially challenging, considering the risk of fetal exposure to ARVs, vis-à-vis risk of transmission. DHHS and WHO European region guidelines suggest that the decision to defer ART initiation until after the first trimester should be based on disease progression in the mother including assessment of CD4 count, viral load, and risk of opportunistic infection.

The recommendations regarding use of efavirenz (EFV) as part of ART are conflicting. DHHS does not approve initiation of EFV based ART in the first trimester, compared to BHIVA who note that there is insufficient evidence to recommend the avoidance of EFV in the first trimester of pregnancy. WHO guidelines for the European region have considered EFV as one of the options for substituting NVP in eligible women with CD4 $>250 / \mathrm{cmm}$.

\section{Choice of drugs and timing of ARV prophylaxis}

All guidelines recommend provision of cARV during antepartum (starting from second trimester), intrapartum periods, and during BF. An additional component of intrapartum care, intravenous azidothymidine (AZT), is 


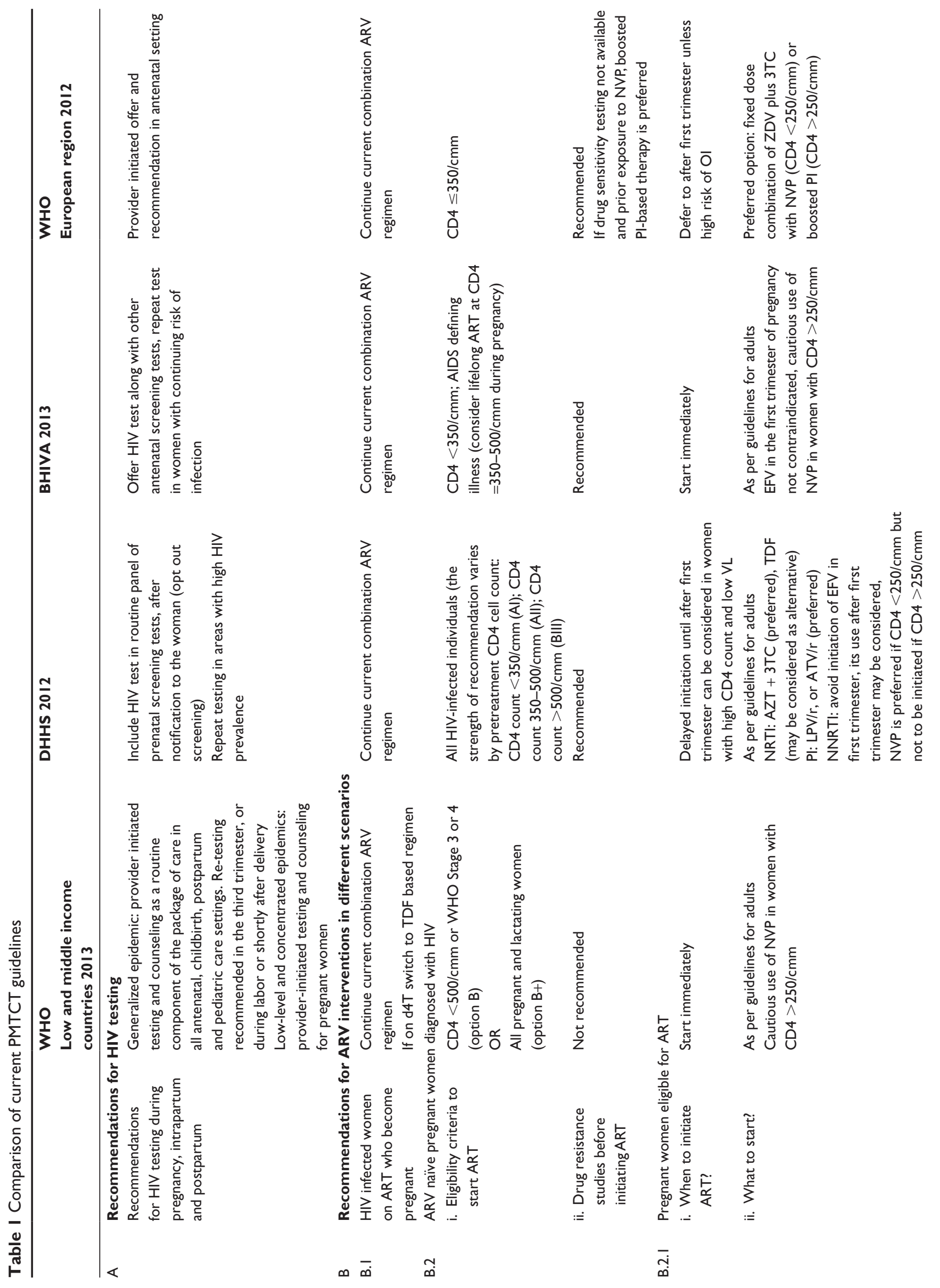



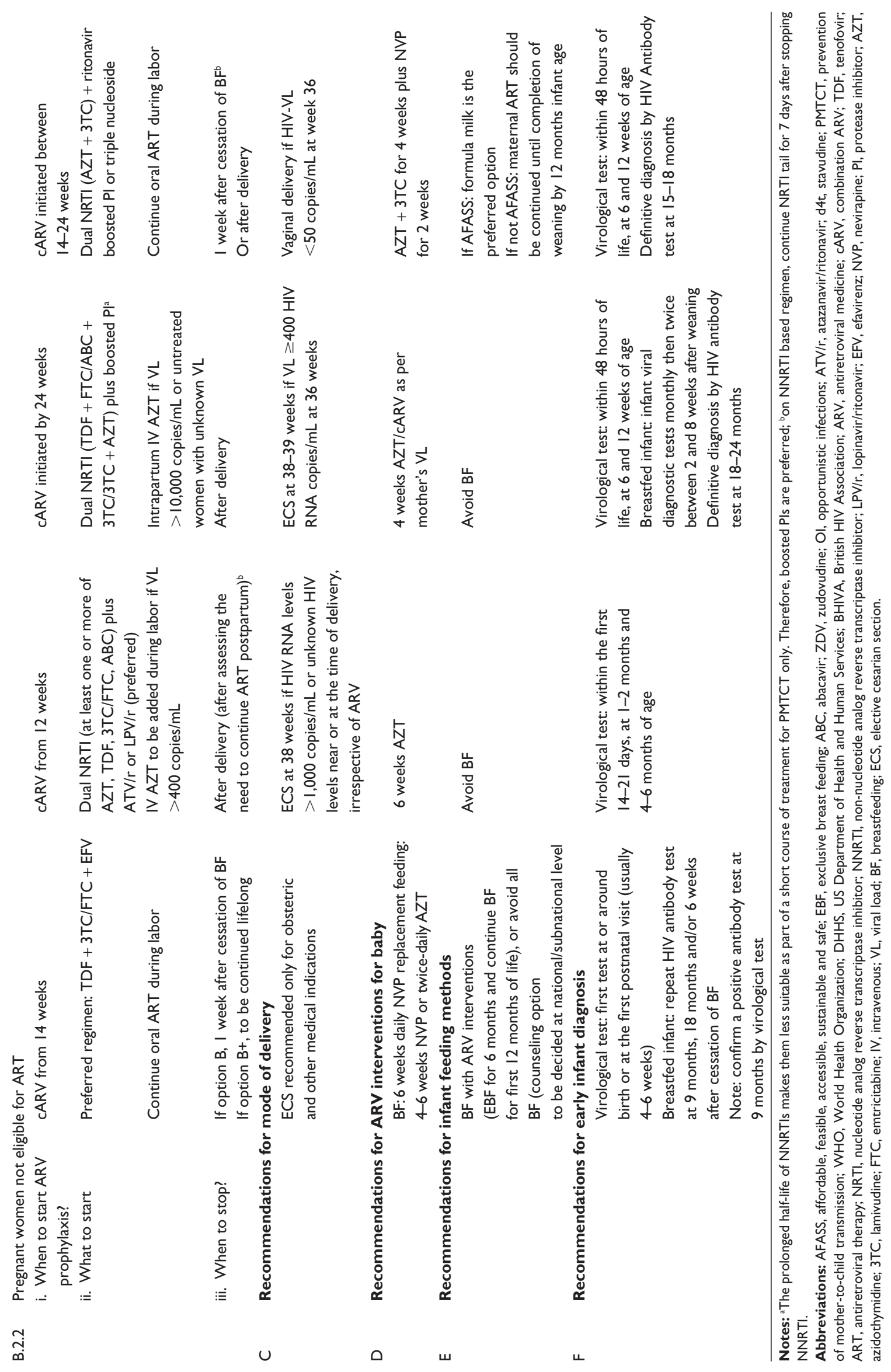
recommended by DHHS and BHIVA guidelines in women with high viral load. Continuation of ARVs until cessation of $\mathrm{BF}$ is recommended in guidelines for developing countries.

Apart from the efficacy of the regimen, the WHO guidelines for LMIC now give consideration to operational issues such as programmatic ease of using a simplified regimen that is harmonized with nonpregnant adults. These guidelines recommend the use of a fixed dose combination of tenofovir (TDF) with lamivudine (3TC), or emtricitabine (FTC) with EFV during pregnancy, and BF as the preferred choice. ${ }^{12}$ The most recent guidelines do not recommend AZT monotherapy to mother and extended NVP to BF infants. ${ }^{12}$

Comparison of these guidelines shows consistency across the guidelines as far as the use of a nucleotide analog reverse transcriptase inhibitors (NRTI) backbone. Major differences are seen with regards to the choice of a third ARV drug. This is mainly due to safety concerns of non-nucleotide analog reverse transcriptase inhibitors (NNRTI). WHO guidelines for LMIC approve EFV based regimens for PMTCT. Protease inhibitor (PI) based regimens remain the protocol of choice for guidelines for developed countries (Table 1).

\section{Mode of delivery and care during the intrapartum period}

Guidelines for LMIC recommend the use of elective cesarean section (ECS) only in the presence of obstetric indications as opposed to all developed countries where ECS is recommended in women with detectable viral load.

With regards to management of labor, WHO 2013 as well as DHHS guidelines recommend wherever possible to avoid the use of invasive instrumentation such as scalp electrodes and blood sampling, amniotomy, instrumental delivery with vacuum/forceps and episiotomy. However the BHIVA guidelines conclude that studies done in the pre-Highly Active Antiretroviral Therapy (HAART) era do not support that these procedures in fact increase the risk. Unfortunately this has not been studied subsequent to the introduction of HAART. As such BHIVA recommends that labor among HIV-infected women be managed as any other woman following national guidelines, in particular where the viral load is suppressed to $<50$ HIV RNA copies/mL. An exception to this is in the case of spontaneous rupture of membranes (ROM) at term where management will depend on the maternal viral load. Induction of labor for those with $<50$ HIV RNA copies/mL is recommended immediately with a very low threshold for management of intra-partum fever due to the increased risk of transmission seen with chorioamnionitis. For those with higher viral loads immediate C-section is recommended. ${ }^{16}$ Additionally it is noted that HIV itself is not a reason to require increased intrapartum fetal monitoring as no increased risk of hypoxia or sepsis has been seen. ${ }^{16}$ During postpartum care, $\mathrm{WHO}^{12}$ encourages washing of blood from the newborn and noninvasive suctioning of nasogastric secretions as a means to reduce risk of transmission, however WHO European region guidelines ${ }^{17}$ suggest that current evidence does not support that any special cleansing methods are beneficial, instead recommending routine care.

\section{Infant feeding practices}

Complete avoidance of BF eliminates risk of MTCT through breast milk. At the same time, it increases risk of infant morbidity in settings with limited access to safer alternatives and poor child care facilities. ${ }^{19}$ Striking a balance between HIV-free survival and eliminating the risk of HIV through BF in such settings becomes challenging. Current WHO guidelines for LMIC recommend exclusive BF for the first 6 months of life, the introduction of appropriate complementary foods thereafter, and the continuation of BF until 12 months of age. BF should only be stopped once a nutritionally adequate and safe diet without breast-milk can be provided. The mother should receive cARV regimen during this entire period of BF. This recommendation is based on evidence that the maximum benefit of BF in preventing mortality from diarrhea, pneumonia, and malnutrition is seen in the first 12 months of life ${ }^{12}$ and that the risk of transmitting HIV to infants through BF is low in the presence of ARV drugs. Due to programmatic difficulties, the option of providing ARVs to HIV exposed babies throughout BF is no longer recommended.

Considering the residual risk of transmission in spite of ARV coverage during $\mathrm{BF}^{20,21}$ and the availability of acceptable, feasible, affordable, sustainable, and safe alternatives to breast milk, guidelines for developed countries consistently recommend avoidance of BF in HIV-infected women.

\section{Implications of different PMTCT protocols}

Combination ARV regimens recommended in current PMTCT guidelines are proven to be most efficacious in reducing MTCT. However, their impact on maternal deaths attributed to HIV/AIDS is yet to be seen. There will also be concerns about potential adverse pregnancy outcomes (APOs) and the safety versus toxicity of cARV during pregnancy and issues related to drug resistance in mother or 
babies acquiring breakthrough infections. ${ }^{22}$ As the coverage of cARV in HIV-infected pregnant women increases, these concerns will have larger implications, especially in high HIV burden countries.

\section{Maternal mortality}

As per current estimates, HIV attributed maternal mortality ratio (15 per 100,000) seen in developing regions is higher than in developed regions (2 per 100,000). ${ }^{23}$ In 2010, 37,000 women died due to AIDS related causes during pregnancy, of which 33,000 were from developing countries. Sub-Saharan Africa was the worst affected region of all. ${ }^{24}$ Studies from South Africa have reported that HIV associated coinfections such as TB, pneumonia, and meningitis are a major cause of death among HIV-infected mothers. ${ }^{25}$ While higher maternal mortality is seen in women with low CD4 counts, the risk of mortality among HIV-infected women was significantly higher compared to HIV uninfected women irrespective of CD4 count. ${ }^{26}$

Wider availability of ART during the last few years does not appear to have had much impact on maternal mortality in women living with HIV. Longitudinal population-based HIV/AIDS data from the African network shows that there has been no significant reduction in maternal mortality during the post HAART era. However cARV based PMTCT interventions were not implemented at any of these study sites during this period. ${ }^{27}$ Many national programs from developing countries have adopted cARV regimens only recently. For example, Malawi rolled out option B+ (lifelong ART to pregnant and BF women) in 2011. Though it is premature to know the actual impact of these changes, it is expected that with increased access to efficacious ARV regimens during pregnancy, HIV related maternal mortality in these regions will reduce. This is corroborated by the findings from an observational study based on a cohort of pregnant women followed in a public health program in Malawi and Mozambique which documents the benefit of using extended antenatal cARV ( $>90$ days) in reducing maternal mortality in HIV-infected women irrespective of their CD4 counts. ${ }^{28}$

\section{APOs}

The evidence on risk of APOs with the use of combination ARVs during pregnancy is conflicting. A combined analysis of data from three observational studies from the USA and Europe showed that HAART was associated with a 1.5-fold increased odds of preterm delivery compared with dual-therapy. ${ }^{29}$ Additionally HAART associated increased risk for preterm delivery when compared to monotherapy was seen in only two of three cohorts (European Collaborative Study and National Study of HIV in Pregnancy and Childhood, but not in the Pediatric Spectrum of HIV Disease project). Authors attribute heterogeneity in the association to differences in populations and data collected. Similar heterogeneity was seen in several other studies from developed ${ }^{30-32}$ and developing ${ }^{28,33-36}$ countries that compared HAART (PI based or non PI based) during pregnancy with no therapy/monotherapy/dual-therapy. Comparisons between PI and non PI based combination ARV regimens have also shown conflicting findings. Secondary data analysis of a randomized control trial from Botswana reported a small increase in risk of preterm birth with use of PI based regimens as compared to 3NRTI begun at 26-34 weeks of pregnancy in HIV-infected women with CD4 $>200 / \mathrm{cmm}^{36}$ This was in contrast to findings from a study of a prospective cohort of women that found no higher risk of preterm birth or low birth weight with use of PI based cART as compared to non PI based cART. ${ }^{32}$

Townsend et al estimated the risk-benefit ratio for HAART in pregnancy based on MTCT and preterm delivery rates from the UK and Ireland surveillance data collected through the National Study of HIV in Pregnancy and Childhood. ${ }^{37}$ They estimated that for every $100 \mathrm{HIV}$ transmissions prevented through the use of HAART (rather than monotherapy), 63 additional preterm deliveries would occur, including 23 at $<32$ weeks gestation. The authors note that interpretation of these ratios is context-dependent and requires additional information about morbidity, mortality and costs associated with the outcomes.

In addition to HAART associated APOs, other factors such as maternal malnutrition also increase the risk of APOs. In a study among rural HIV-infected women from Uganda initiating cART, anemia and inadequate gestational weight gain were commonly seen and were associated with APOs. ${ }^{38}$

In regions with a high burden of HIV, APO in HIVinfected women may pose an additional burden to health facilities. Health care systems will need to be prepared to meet these emerging needs. Provision of cART as a part of comprehensive maternal and child health care will be a key strategy to improve pregnancy related outcomes in HIVinfected women in LMIC.

\section{Safety and toxicity of triple drug regimens during pregnancy and BF}

Extensive information is now available about the toxicity profile of different ARVs in adults and how these can be monitored. However there are additional concerns 
regarding certain ARVs during pregnancy. Information on ARVs such as EFV and TDF during pregnancy is limited as compared to the long experience that AZT, NVP, and LPV/r (lopinavir/ritonavir) have had.

Although available data suggest that the concerns about risk of neural tube defects in babies with use of EFV during pregnancy are unfounded, ${ }^{39}$ continued surveillance is warranted with increasing coverage of PMTCT programs. Current antiretroviral registry ${ }^{40}$ data does not indicate an increased risk of birth defects with EFV during the first or second trimesters. Neurological effects and depression are known side effects of EFV. The additional impact of EFV on depression commonly associated with pregnancy and the postpartum period is not known. Although decreased bone mineral density has been observed in children with chronic use of TDF, in utero exposure to TDF does not appear to affect growth in HIV exposed children. ${ }^{41,42}$ Bone and renal toxicity have been documented in adults put on TDF. Additionally lactating women normally have bone loss during BF and it is not yet known if this is exaggerated with use of TDF during this period. More evidence about TDF toxicity in pregnant and lactating women is needed to determine optimal monitoring during this period.

Severe NVP induced hepatotoxicity is observed among pregnant women with a CD 4 count $>250 / \mathrm{cmm}$. Initiation of NVP is best avoided in pregnant women with high CD4 counts. AZT is known to adversely impact hematologic parameters. A small reduction in hemoglobin in the mother and infant is seen with AZT exposure.

Recognizing the need of further evidence to reduce the uncertainty, WHO recommends that toxicity surveillance systems and additional research should be undertaken by national programs in low and middle income countries. ${ }^{43}$

\section{Drug resistance in pregnant women} with prior exposure to ARVs

Different ARV regimens such as single dose (sd) NVP, short- and long-term AZT with or without sd NVP, and pregnancy limited dual/triple ARVs have been used as PMTCT prophylaxis in various settings. Short-term mono- or dualtherapy ARV regimens are likely to induce resistant mutations. High rates of M184V/I mutation, which confer high level resistance to $3 \mathrm{TC} / \mathrm{FTC}$, were seen among women who received a short course of dual ARVs during pregnancy, ${ }^{44}$ adversely affecting virological response in the mother with subsequent initiation of ART for her own disease. ${ }^{45}$ A metaanalysis among women who received single-dose NVP with or without other antepartum antiretrovirals reported prevalence of NVP resistance as high as $35.7 \%(95 \% \mathrm{CI}$, 23.0-50.6). ${ }^{46}$ A higher risk of virological failure was seen with NNRTI based ART in women with recent exposure to sd $\mathrm{NVP},{ }^{47}$ but this risk diminished with time. ${ }^{48}$ Also, the $\mathrm{TDF}+\mathrm{FTC}+\mathrm{LPV} / \mathrm{r}$ regimen was found to be superior in such women as compared to TDF/FTC/NVP when used subsequently. Very low rates of resistance mutations were seen with the use of pregnancy-limited PI-based combination ARV regimens. ${ }^{44}$

A large number of pregnant women from developing countries have been exposed to mono- or dual-therapy ARV based PMTCT regimens until 2010. ${ }^{49}$ They could be at higher risk of treatment failure when started on NRTI + NNRTI based combination therapy and this also introduces the risk of transmission of drug-resistant virus. ${ }^{22}$ These women might have limited options for future ART. In such situations, close virological monitoring of these women is essential. Some national programs have considered alternate options for such women. For example, the National AIDS Control Organisation (NACO) in India recommends use of a $\mathrm{LPV} / \mathrm{r}$ based regimen in women with previous exposure to NNRTI. ${ }^{50}$

\section{Drug resistance in children with breakthrough infection}

A high proportion of resistant mutations have been observed in HIV-infected children exposed to NNRTI used for PMTCT. However resistance profiles in children exposed to triple drug combination during pregnancy and BF are not well-known. In a study among women initiating HAART $(\mathrm{NVP}+\mathrm{d} 4 \mathrm{~T}$ [stavudine] $+3 \mathrm{TC})$ during the BF period, 11 of 37 infants were detected to have multiclass resistance. ${ }^{51}$ A secondary analysis from Kisumu Breastfeeding Study (KiBS) data assessed emergence of resistance to triple drug maternal ART (ZDV [zudovudine]/3TC and either NVP or nelfinavir [NFV]) given from 34 weeks to 6 months postpartum. Sixteen out of 24 infected infants were found to have mutations conferring resistance to $3 \mathrm{TC}$ and NVP. ${ }^{52}$ Limited data exist about resistance profiles in HIV-infected children exposed to regimens containing TDF and EFV given during pregnancy and BF. There is little penetration of TDF through breast milk and it is not known if feeding will increase the risk of resistance to EFV and 3TC in infants.

The choice of an optimal regimen in children with breakthrough infections will be a critical issue in the future. Currently LPV/r-based regimens are the preferred choice for treating young HIV-infected children. The efficacy of this regimen in children with multiclass resistance is 
not known. More evidence in this regard is needed to inform guidelines for LMIC where access to drug resistance testing is minimal.

\section{Translating scientific knowledge into action: coverage of PMTCT services}

Now that new guidelines are available, translating scientific advancement into field level action to achieve maximum gains in reducing MTCT will be the challenge faced by service providers. Heterogeneity exists in the HIV epidemic as well as in the coverage of PMTCT services across countries and regions. Coverage is highest in Eastern and Central Europe and the Caribbean (more than 90\%), while coverage is much lower in Asia and the Pacific and the Middle East and North Africa (less than 20\%). ${ }^{8}$

While developed countries have long maintained near universal coverage of PMTCT services, providing such coverage in developing countries where more than $90 \%$ of HIV transmission to babies occurs has been a difficult task. For effective PMTCT programs, it is essential that women complete the entire cascade of PMTCT services.

Several reviews published in the past 2 years suggest an unacceptably high proportion of loss to follow up (LTFU) of HIV-infected women at different stages of the cascade of PMTCT services. ${ }^{53-56}$ The LTFU of women between antenetal clinic (ANC) registration and delivery or of women who do not receive any ARV prophylaxis is reported to be approximately $50 \%$ in a review by Sibanda et al,${ }^{54}$ and Tudor Car et al, ${ }^{55}$ and $30 \%$ by Wettstein et al. ${ }^{56}$ The range of LTFU of infants from PMTCT programs before undergoing HIV testing was reported between $21 \%-36 \%$ in these studies. These reviews also noted significant LTFU of HIV-infected children who were linked to HIV care, with obvious detrimental consequences. The inability to retain women in PMTCT programs has major implications not only for preventing HIV transmission to the baby, but also for providing comprehensive HIV care to an infected women, her infected partner or child, and to the provision of family planning services (affecting both Prongs 2 and 4 of the WHO approach). ${ }^{57}$

HIV testing during pregnancy, which is considered as the first step in the cascade of PMTCT services, is still beyond the reach of many pregnant women in developing countries. ${ }^{8}$ Personal barriers to testing include poor knowledge about HIV, young maternal age, lack of education and family support, denial of risk, cultural considerations and fear of stigma. ${ }^{53,54,56}$ Systemic barriers include lack of access to timely ANC care resulting in late presentation, non-facility deliveries, shortage of trained staff and test kits, cost and distance issues and concerns around privacy and disclosure issues such as inadequate counseling spaces. $^{53-56}$

Among the approaches for getting women to access services for antenatal care, ideally in a setting where there is integration of her care with other family health services, ${ }^{57}$ community involvement seems to play a key role. ${ }^{58-61}$ While a recent review of published literature on community-based and community-oriented strategies demonstrate increased effectiveness of the PMTCT programs ${ }^{62}$ there is also recognition that there is no one-size-fits-all for community engagement. ${ }^{63}$ Effective and meaningful community engagement requires consideration for epidemiological, socio-cultural and health systems related factors.

The next step in the cascade involves initiating ART. In countries with a high burden of HIV such as the Democratic Republic of Congo, Angola, Chad, Ethiopia and Nigeria, less than $50 \%$ of the pregnant women received antiretroviral preventive services in 2012. ${ }^{8}$ Barriers to women receiving ART include personal compliance issues as well as systemic issues of human, material, and financial resources. ${ }^{55}$ Potential facilitators may lie in the ability to find simplified methods to diagnose and evaluate women's needs and eligibility for cART. $^{57}$

Once women are linked to care facilities, continued access to PMTCT services has been shown to be improved not just by providing cART but also by involving male partners (Prong 3 and Prong 4 of WHO approach). ${ }^{56,58}$ Lack of male involvement has been identified as one of the barriers in implementing effective PMTCT programs. While the benefits of involving men in PMTCT programs for preventing HIV transmission within couples as well as increasing the uptake of PMTCT services ${ }^{64}$ has been shown and strategies to involve men in $\mathrm{PMTCT}^{65}$ have been documented, there is limited research evidence on the effectiveness of male involvement in improving PMTCT outcomes. ${ }^{66,67}$

The integration of PMTCT services within sexual and reproductive health services is another important programmatic strategy that has been suggested for improving the effectiveness of PMTCT program outcomes (Prong 1 and Prong 2 of WHO approach). ${ }^{57,61}$ There are several compelling reasons for taking this approach starting with the fact that the target groups for both these services are the same. From the perspective of the recipient, who is unlikely to separate her sexual behaviors and the consequences of those into neat boxes marked "HIV", "pregnancy", and "other STIs", the health messages and services delivered are often similar, 
making their delivery more efficient. Such convergence could also have logistical benefits for programs in terms of the ease of dealing with issues of procurement and program management and by decreasing the resources required. It has also been suggested that the provision of strong family planning services and contraception to avoid unplanned pregnancies may even have a greater impact on preventing MTCT than HIV testing and diagnosis. ${ }^{60}$ Although not much data are currently available regarding the impact of integration of PMTCT and other RCH services, a Cochrane review by Tudor Car et $\mathrm{al}^{68}$ did not find sufficient evidence to make definitive conclusions about the effectiveness of integration of these interventions with other health services compared to providing them as stand-alone services. Further research on intervention coverage, service uptake, quality of care and health outcomes and the optimal integration modality is urgently needed to assess the effect of integrating perinatal PMTCT interventions with other health services. Another potential facilitator to overcome the challenge of providing ART includes the role of simplified PMTCT protocols. Several reviews have addressed this, and its relationship with LTFU, suggesting that it may reduce the barriers to accessing services. ${ }^{53,54}$ An emerging approach in PMTCT in developing countries is to provide cART (option $\mathrm{B}+$ ). While provision of cART can have the effect of potentially reducing individual level barriers ${ }^{53}$ due to improved contacts with the health systems/counselors and due to improved monitoring (CD4 testing/adherence monitoring), other challenges will emerge mainly related to the health systems requirements needed to support B+ including supply chain and ongoing clinical monitoring for patients. Delivery of the $\mathrm{B}+$ guidelines will therefore require health systems strengthening. ${ }^{61}$ Figure 2 shows the potential effect of cART on barriers in accessing PMTCT services.

\section{Summary}

Rapid changes are occurring in the PMTCT scenario especially in developing countries. The increasing similarity in the guidelines regarding PMTCT between developed and developing countries is opening up the opportunity to envision the elimination of pediatric HIV. Guidelines now show changes in ARV regimes from monotherapy to cARV and from short-term prophylaxis to lifelong treatment for women. Issues related not only to the practical implementation of these changes, but to safety and toxicities of ARVs, drug resistance in mothers and in children with breakthrough infections, and adherence to ARVs during and after the postpartum period will become important questions in the context of PMTCT that will need to be addressed. Furthermore, as more efficacious drug regimens are adopted for PMTCT, the effectiveness of PMTCT programs will now become determined by the coverage of services and the recruitment and retention of pregnant women in the cascade. Both barriers and facilitators have been documented which impact the uptake of services at several steps across the cascade of continuum of care of HIV-infected women and children. To expand services and provide an effective, comprehensive approach to improve program effectiveness, there is a need to harmonize efforts to address barriers whilst considering contextual factors.

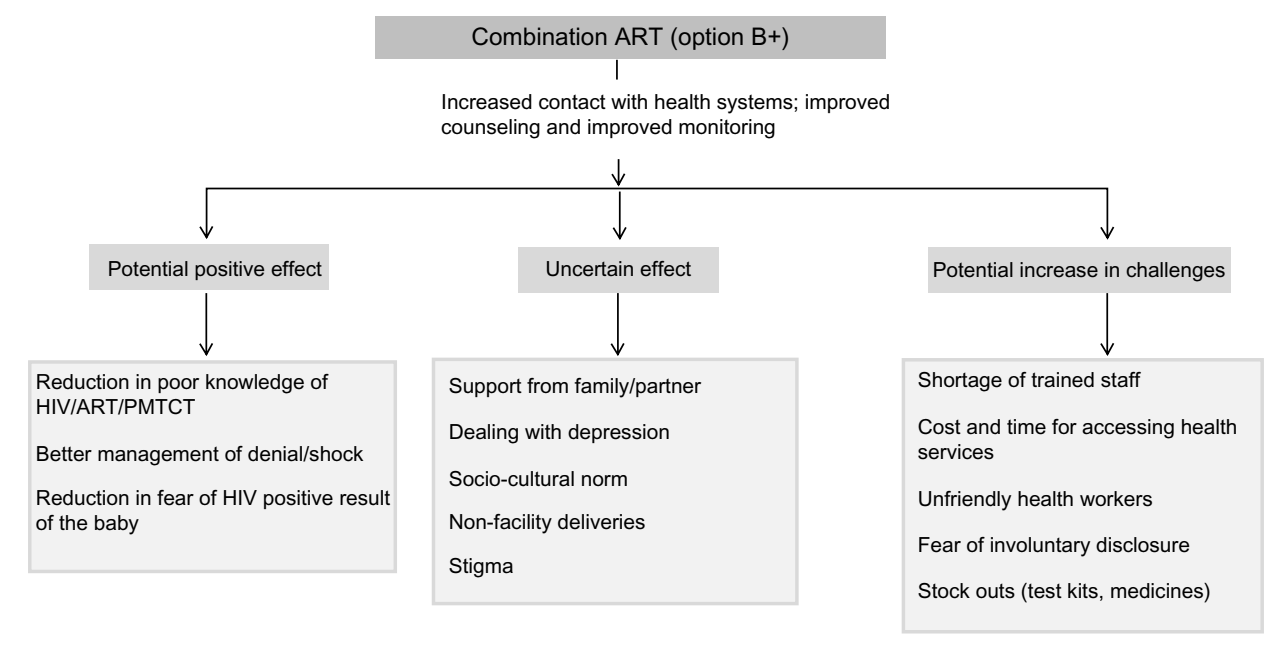

Figure 2 Possible effect of combination antiretroviral therapy (cART) on barriers to accessing continuing care due to increased number of visits to the counselor and improved monitoring.

Abbreviation: PMTCT, prevention of mother-to-child transmission. 


\section{Disclosure}

The authors report no conflicts of interest in this work.

\section{References}

1. Connor EM, Sperling RS, Gelber R, et al. Reduction of maternal-infant transmission of human immunodeficiency virus type 1 with zidovudine treatment. Pediatric AIDS Clinical Trials Group Protocol 076 Study Group. N Engl J Med. 1994;331(18):1173-1180.

2. Darak S, Panditrao M, Parchure R, Kulkarni V, Kulkarni S, Janssen F. Systematic review of public health research on prevention of mother-to-child transmission of HIV in India with focus on provision and utilization of cascade of PMTCT services. BMC Public Health. 2012;12:320.

3. Cooper ER, Charurat M, Mofenson L, et al. Combination antiretroviral strategies for the treatment of pregnant HIV-1-infected women and prevention of perinatal HIV-1 transmission. J Acquir Immune Defic Syndr. 2002;29(5):484-494.

4. Global HIV/AIDS Response. Epidemic update and health sector progress towards Universal Access. Progress Report 2011. UNAIDS; 2011. Available from: http://www.unaids.org/en/media/unaids/ contentassets/documents/unaidspublication/2011/20111130_ua_ report_en.pdf. Accessed May 21, 2014.

5. Global plan towards the elimination of new HIV infections among children by 2015 and keeping their mothers alive. 2011-2015. UNAIDS; 2011. Available from: http://www.unaids.org/en/media/ unaids/contentassets/documents/unaidspublication/2011/20110609_ jc2137_global-plan-elimination-hiv-children_en.pdf. Accessed May 21, 2014.

6. Mahy M, Stover J, Kiragu K, et al. What will it take to achieve virtual elimination of mother-to-child transmission of HIV? An assessment of current progress and future needs. Sex Transm Infect. 2010;86(Suppl 2): ii48-ii55.

7. Hairston AF, Bobrow EA, Pitter CS. Towards the Elimination of Pediatric HIV: Enhancing Maternal, Sexual, and Reproductive Health Services. International Journal of MCH and AIDS. 2012;1(1):6-16.

8. Global report. UNAIDS report on the global AIDS epidemic 2013. UNAIDS; 2013. Available from: http://www.unaids.org/en/media/ unaids/contentassets/documents/epidemiology/2013/gr2013/UNAIDS_ Global_Report_2013_en.pdf. Accessed May 21, 2014.

9. Ciaranello AL, Perez F, Keatinge J, et al. What will it take to eliminate pediatric HIV? Reaching WHO target rates of mother-to-child HIV transmission in Zimbabwe: a model-based analysis. PLoS Med. 2012;9(1):e1001156.

10. Siegfried N, van der Merwe L, Brocklehurst P, Sint TT. Antiretrovirals for reducing the risk of mother-to-child transmission of HIV infection. Cochrane Database Syst Rev. 2011;(7):CD003510.

11. Jamieson DJ, Chasela CS, Hudgens MG, et al. Maternal and infant antiretroviral regimens to prevent postnatal HIV-1 transmission: 48-week follow-up of the BAN randomised controlled trial. Lancet. 2012;379(9835):2449-2458.

12. Consolidated guidelines on the use of antiretroviral drugs for treating and preventing HIV infection. Recommendation for a public health approach. World Health Organization; 2013. Available from: http:// apps.who.int/iris/bitstream/10665/85321/1/9789241505727_eng.pdf. Accessed May 21, 2014.

13. Guidance on provider-initiated HIV testing and counselling in health facilities. World Health Organization; 2007. Available from: http://www. unicef.org/aids/files/PITCGuidance2007_Eng.pdf. Accessed May 21, 2014.

14. Antiretroviral drugs for treating pregnant women and preventing HIV infection in infants in resource limited settings. Towards universal access. Recommendations for a public health approach. World Health Organization; 2006. Available from: http://www.who.int/hiv/pub/ guidelines/WHOPMTCT.pdf. Accessed May 21, 2014.
15. Antiretroviral drugs for treating pregnant women and preventing HIV infection in infants: recommendations for a public health approach. 2010 version. World Health Organization; 2010. Available from: http://whqlibdoc.who.int/publications/2010/9789241599818_eng.pdf. Accessed May 21, 2014.

16. British HIV Association guidelines for the management of HIV infection in pregnant women 2012. British HIV Association; 2012. HIV Medicine. 2012;13(Suppl 2):87-157. Available from: http://www.bhiva. org/documents/Guidelines/Pregnancy/2012/hiv1030_6.pdf. Accessed May 21, 2014.

17. Prevention of HIV transmission from HIV-infected mothers to their infants. Optimizing clinical management and effective interventions towards eliminating new paediatric HIV infections. Clinical Protocol for the WHO European Region (2012 revision). World Health Organization; 2012. Available from: http://www.euro.who.int/_data/ assets/pdf_file/0007/159973/PMTCT_20120302.pdf. Accessed May 21, 2014.

18. Recommendations for Use of Antiretroviral Drugs in Pregnant HIV-1-Infected Women for Maternal Health and Interventions to Reduce Perinatal HIV Transmission in the United States. 2011. Available from: http://aidsinfo.nih.gov/contentfiles/lvguidelines/ PerinatalGL.pdf. Accessed May 21, 2014.

19. Horvath T, Madi BC, Iuppa IM, Kennedy GE, Rutherford G, Read JS. Interventions for preventing late postnatal mother-to-child transmission of HIV. Cochrane Database Syst Rev. 2009;(1):CD006734.

20. Recommendations for Use of Antiretroviral Drugs in Pregnant HIV-1Infected Women for Maternal Health and Interventions to Reduce Perinatal HIV Transmission in the United States. 2010. Available from: http://www.antimicrobe.org/h04c.files/history/PerinatalGL.pdf. Accessed May 21, 2014.

21. Taylor GP, Anderson J, Clayden P, et al. British HIV Association and Children's HIV Association position statement on infant feeding in the UK 2011. HIV Med. 2011;12(7):389-393.

22. Rongkavilit C, Asmar BI. Advances in prevention of mother-to-child HIV transmission: the international perspectives. Indian J Paediatr. 2011;78:192-204.

23. Trends in Maternal Mortality: 1990 to 2010. WHO, UNICEF, UNFPA and The World Bank estimates. World Health Organization; 2012. Available from: https://www.unfpa.org/webdav/site/global/shared/ documents/publications/2012/Trends_in_maternal_mortality_A4-1. pdf. Accessed May 21, 2014.

24. A progress report on the Global Plan towards the elimination of new HIV infections among children by 2015 and keeping their mothers alive. UNAIDS; 2012. Available from: http://www.unaids.org/en/ media/unaids/contentassets/documents/unaidspublication/2012/ JC2385_ProgressReportGlobalPlan_en.pdf. Accessed May 21, 2014.

25. Moodley J, Pattinson RC, Baxter C, Sibeko S, Abdool Karim Q. Strengthening HIV services for pregnant women: an opportunity to reduce maternal mortality rates in Southern Africa/sub-Saharan Africa. BJOG. 2011;118(2):219-225.

26. Hargrove JW, Humphrey JH, ZVITAMBO Study Group. Mortality among HIV-positive postpartum women with high CD4 cell counts in Zimbabwe. AIDS. 2010;24(3):F11-F14.

27. Zaba B, Calvert C, Marston M, et al. Effect of HIV infection on pregnancy-related mortality in sub-Saharan Africa: secondary analyses of pooled community-based data from the network for Analysing Longitudinal Population-based HIV/AIDS data on Africa (ALPHA). Lancet. 2013;381(9879):1763-1771.

28. Marazzi MC, Palombi L, Nielsen-Saines K, et al. Extended antenatal use of triple antiretroviral therapy for prevention of mother-to-child transmission of HIV-1 correlates with favorable pregnancy outcomes. AIDS. 2011;25(13):1611-1618.

29. Townsend C, Schulte J, Thorne C, et al. Antiretroviral therapy and preterm delivery-a pooled analysis of data from the United States and Europe. BJOG. 2010;117(11):1399-1410. 
30. Grosch-Woerner I, Puch K, Maier RF, et al. Increased rate of prematurity associated with antenatal antiretroviral therapy in a German/Austrian cohort of HIV-1-infected women. HIV medicine. 2008;9(1):6-13.

31. Lopez M, Figueras F, Hernandez S, et al. Association of HIV infection with spontaneous and iatrogenic preterm delivery: effect of HAART. AIDS. 2012;26(1):37-43.

32. Patel K, Shapiro DE, Brogly SB, et al. Antenatal Protease Inhibitor use and Risk of Preterm Birth among HIV-infected Women Initiating Antiretrovirals during Pregnancy. J Infect Dis. 2010;201(7): 1035-1044

33. Chen JY, Ribaudo HJ, Souda S, et al. Highly active antiretroviral therapy and adverse birth outcomes among HIV-infected women in Botswana. J Infect Dis. 2012;206(11):1695-1705.

34. Darak S, Darak T, Kulkarni S, et al. Effect of highly active antiretroviral treatment (HAART) during pregnancy on pregnancy outcomes: experiences from a PMTCT program in western India. AIDS Patient Care STDs. 2013;27(3):163-170.

35. Machado ES, Hofer CB, Costa TT, et al. Pregnancy outcome in women infected with HIV-1 receiving combination antiretroviral therapy before versus after conception. Sex Transm Infect. 2009;85(2):82-87.

36. Powis KM, Kitch D, Ogwu A, et al. Increased risk of preterm delivery among HIV-infected women randomized to protease versus nucleoside reverse transcriptase inhibitor-based HAART during pregnancy. J Infect Dis. 2011;204(4):506-514.

37. Townsend CL, Cortina-Borja M, Peckham CS, Tookey PA. Antiretroviral therapy and premature delivery in diagnosed HIVinfected women in the United Kingdom and Ireland. AIDS. 2007;21(8): 1019-1026.

38. Young S, Murray K, Mwesigwa J, et al. Maternal nutritional status predicts adverse birth outcomes among HIV-infected rural Ugandan women receiving combination antiretroviral therapy. PloS One. 2012;7(8): e41934.

39. Ford N, Calmy A, Mofenson L. Safety of efavirenz in the first trimester of pregnancy: an updated systematic review and meta-analysis. AIDS. 2011;25(18):2301-2304.

40. Antiretroviral Pregnancy Registry. Antiretroviral Pregnancy Registry International Interim Report for January 1, 1989 through July 31, 2013. Available from: http://www.apregistry.com/forms/interim_report.pdf. Accessed May 21, 2014.

41. Wang L, Kourtis AP, Ellington S, Legardy-Williams J, Bulterys M. Safety of tenofovir during pregnancy for the mother and fetus: a systematic review. Clin Infect Dis. 2013;57(12):1773-1781.

42. Chi BH, Stringer JS, Moodley D. Antiretroviral Drug Regimens to Prevent Mother-To-Child Transmission of HIV: A Review of Scientific, Program, and Policy Advances for Sub-Saharan Africa. Curr HIV/AIDS Rep. 2013;10(2):124-133.

43. Surveillance of antiretroviral toxicity HIV/AIDS programme surveillance of antiretroviral drug toxicity during pregnancy and breastfeeding. World Health Organization; 2013. Available from: http:// apps.who.int/iris/bitstream/10665/91578/1/WHO_HIV_2013.124_eng. pdf. Accessed May 21, 2014.

44. Paredes R, Lalama CM, Ribaudo HJ, et al. Pre-existing minority drugresistant HIV-1 variants, adherence, and risk of antiretroviral treatment failure. Pre-existing minority drug-resistant HIV-1 variants, adherence, and risk of antiretroviral treatment failure. J Infect Dis. 2010;201(5): 662-671.

45. Coffie PA, Ekouevi DK, Chaix ML, et al. Maternal 12-month response to antiretroviral therapy following prevention of mother-to-child transmission of HIV type 1, Ivory Coast, 2003-2006. Clin Infect Dis. 2008;46(4):611-621.

46. Arrivé E, Newell ML, Ekouevi DK, et al. Prevalence of resistance to nevirapine in mothers and children after single-dose exposure to prevent vertical transmission of HIV-1: a meta-analysis. Int J Epidemiol. 2007;36(5):1009-1021.

47. Lockman S, Shapiro RL, Smeaton LM, et al. Response to antiretroviral therapy after a single, peripartum dose of nevirapine. $N$ Engl J Med. 2007;356(2):135-147.
48. Stringer JS, McConnell MS, Kiarie J, et al. Effectiveness of non-nucleoside reverse-transcriptase inhibitor-based antiretroviral therapy in women previously exposed to a single intrapartum dose of nevirapine: a multi-country, prospective cohort study. PLoS Medicine. 2010;7(2):e1000233.

49. PMTCT strategic vision 2010-2015. Preventing mother-to-child transmission of HIV to reach the UNGASS and Millennium Development Goals. World Health Organization; 2010. Available from: http://whqlibdoc.who.int/publications/2010/9789241599030_eng.pdf. Accessed May 21, 2014.

50. National Guidelines for Prevention of Parent-to-Child Transmission (PPTCT) of HIV. NACO; 2012. Available from: http://www. naco.gov.in/upload/Publication/Basic\%20Services/National\%20 Guidelines\%20for\%20PPTCT_01_05_2013.pdf. Accessed May 21, 2014.

51. Fogel J, Li Q, Taha TE, et al. Initiation of antiretroviral treatment in women after delivery can induce multiclass drug resistance in breastfeeding HIV-infected infants. Clin Infect Dis. 2011;52(8): 1069-1076.

52. Zeh C, Weidle PJ, Nafisa L, et al. HIV-1 drug resistance emergence among breastfeeding infants born to HIV-infected mothers during a single-arm trial of triple-antiretroviral prophylaxis for prevention of mother-to-child transmission: a secondary analysis. PLoS Medicine. 2011;8(3):e1000430.

53. Gourlay A, Birdthistle I, Mburu G, Iorpenda K, Wringe A. Barriers and facilitating factors to the uptake of antiretroviral drugs for prevention of mother-to-child transmission of HIV in sub-Saharan Africa: a systematic review. J Int AIDS Soc. 2013;16(1):18588.

54. Sibanda EL, Weller IV, Hakim JG, Cowan FM. The magnitude of loss to follow-up of HIV-exposed infants along the prevention of mother-to-child HIV transmission continuum of care: a systematic review and meta-analysis. AIDS. 2013;27(17):2787-2797.

55. Tudor Car L, Brusamento S, Elmoniry H, et al. The uptake of integrated perinatal prevention of mother-to-child HIV transmission programs in low- and middle-income countries: a systematic review. PloS One. 2013;8(3):e56550.

56. Wettstein C, Mugglin C, Egger M, et al. Missed opportunities to prevent mother-to-child-transmission: systematic review and meta-analysis. AIDS. 2012;26(18):2361-2373.

57. Kellerman SE, Ahmed S, Feeley-Summerl T, et al. Beyond prevention of mother-to-child transmission: keeping HIV-exposed and HIV-positive children healthy and alive. AIDS. 2013;27(Suppl 2):S225-S233.

58. Larsson EC, Thorson AE, Pariyo G, et al. Missed Opportunities: barriers to HIV testing during pregnancy from a population based cohort study in rural Uganda. PloS One. 2012;7(8):e37590.

59. Khumalo-Sakutukwa G, Morin SF, Fritz K, et al. Project Accept (HPTN 043): A Community-Based Intervention to Reduce HIV Incidence in Populations at Risk for HIV in Sub-Saharan Africa and Thailand. J Acquir Immune Defic Syndr. 2008;49(4):422-431.

60. Govender T, Coovadia H. Eliminating mother to child transmission of HIV-1 and keeping mothers alive: Recent progress. J Infect. 2014;68(Suppl 1):S57-S62.

61. Chi BH, Adler MR, Bolu O. Progress, challenges, and new opportunities for the prevention of mother-to-child transmission of HIV under the US President's Emergency Plan for AIDS Relief. $J$ Acquir Immune Defic Syndr. 2012;60(Suppl 3):S78-S87.

62. Marcos Y, Phelps BR, Bachman G. Community strategies that improve care and retention along the prevention of mother-to-child transmission of HIV cascade: a review. J Int AIDS Soc. 2012;15(Suppl 2):17394

63. Promising practices in community engagement for elimination of new HIV infections among children by 2015 and keeping their mothers alive. UNAIDS; 2012. Available from: http://www.unaids.org/en/media/ unaids/contentassets/documents/unaidspublication/2012/20120628_ JC2281_PromisingPracticesCommunityEngagements_en.pdf. Accessed May 21, 2014. 
64. Male involvement in the prevention of mother-to-child transmission of HIV. World Health Organization; 2012. Available from: http://www.who. int/reproductivehealth/publications/rtis/9789241503679/en/. Accessed May 21, 2014.

65. Orne-Gliemann J, Balestre E, Tchendjou P, et al. Increasing HIV testing among male partners. AIDS. 2013;27(7):1167-1177.

66. Auvinen J, Kylma J, Suominen T. Male involvement and prevention of mother-to-child transmission of HIV in Sub-Saharan Africa: an integrative review. Current HIV Res. 2013;11(2):169-177.
67. Brusamento S, Ghanotakis E, Tudor Car L, van-Velthoven $\mathrm{MH}$, Majeed A, Car J. Male involvement for increasing the effectiveness of prevention of mother-to-child HIV transmission (PMTCT) programmes. Cochrane Database Syst Rev. 2012;10:CD009468.

68. Tudor Car L, van-Velthoven MH, Brusamento S, et al. Integrating prevention of mother-to-child HIV transmission (PMTCT) programmes with other health services for preventing HIV infection and improving HIV outcomes in developing countries. Cochrane Database Syst Rev. 2011;6:CD008741.

\section{Publish your work in this journal}

Research and Reports in Neonatology is an international, peer-reviewed, open access journal publishing original research, reports, editorials, reviews and commentaries on neonatal health. The manuscript management system is completely online and includes a very quick and fair peer-review system. Visit http://www.dovepress.com/testimonials.php to read real quotes from published authors. 